\section{An inflammatory vaccine}

\section{By Lauren Martz, Staff Writer}

Researchers at The University of Texas M.D. Anderson Cancer Center have taken a new approach to DNA cancer vaccines: damaging host tissue to induce an inflammatory response and greater immune recognition of the antigen. ${ }^{1}$ The team hopes its modified idiotype-based vaccine plus an inflammation-inducing myotoxin adjuvant can overcome the poor efficacy of most previously tested DNA vaccines-a clinical trial is already in the planning.

Idiotypes are DNA sequences coding for unique portions of the variable regions of the heavy and light chains of the immunoglobulins expressed on the surface of B cells. Researchers at the National Cancer Institute have idiotype vaccines in Phase II testing to treat lymphoma. ${ }^{2}$

However, DNA vaccination strategies have so far underperformed because the plasmid DNA-based vaccines fail to induce a significant immune response and the process required to develop the patient- and tumor-specific treatments is laborious.

To address these issues, Larry Kwak and colleagues at M.D. Anderson have developed a more streamlined, secondgeneration procedure to create lymphoma vaccines. The key, they think, was fusing the idiotype sequence to a DNA sequence encoding monocyte chemoattractant protein 3 (MCP-3; CCL7) and administering it with a myotoxin adjuvant.

Kwak said the chemokine MCP-3 allows direct targeting of the vaccine to antigen-presenting cells (APCs). The myotoxin induces a local inflammatory response at the injection site, which is expected to help the vaccine come in contact with more cells of the host immune system, thus increasing efficacy and eliciting antitumor memory.

The researchers first compared the effect of cardiotoxin, a myotoxin found in viper venom, with four toll-like receptor (TLR) adjuvants known to increase the potency of vaccine-induced antigen-specific $T$ cell responses. ${ }^{3}$ The vaccine plus cardiotoxin combination was most effective at protecting against tumor challenge.

Studies using different idiotype DNA vaccines and a different myotoxin produced similar results.

To test whether the strategy induced antitumor memory, the researchers took mice rendered tumor-free after an initial treatment with DNA vaccine plus cardiotoxin followed by a cancer challenge and rechallenged them with another lethal dose of lymphoma cells. Of these animals, $80 \%$ were protected compared with only $40 \%$ of control animals receiving vaccine alone.

The group also showed that both humoral- and tumor-specific $\mathrm{T}$ cell immune responses were generated by the method, with both tumor-specific B cells and T cells present. Although both types of immunity were actively involved, only the $\mathrm{T}$ cell response was required for antitumor activity. Depletion of the $\mathrm{T}$ cell population prevented the antitumor effects, but there was no significant change in effect with B cell depletion.

The results were published in Blood.

"Some process optimization will be required, but we're planning a Phase I clinical trial with the DNA fusion vaccine. The clinical protocol has been drafted and it now needs to make its way through the IRB [internal review board]," said Kwak, who is chairman of the Department of Lymphoma at M.D. Anderson.

\section{Vaccine design}

In addition to improved potency, the M.D. Anderson group expects its DNA vaccine to take less time to produce and require less-invasive procedures than previous idiotype vaccines.

Kwak noted that first-generation methods for producing idiotype vaccines require a surgical excision of about $2 \mathrm{~cm}$ from the lymph node. He also said that the process involves producing proteins from the tumor genes, which can be time consuming.

His team's method, he said, "is much more streamlined and less labor intensive. It is also at least as potent as the first-generation version with proof in animal models. In this process, it is only necessary to clone the genes rather than actually make the protein products. You don't need as much starting material-you can do a fine-needle core biopsy, which is much less invasive than the surgical excision."

Kwak also said that using first-generation methods, it could take 4-6 weeks to generate an individualized vaccine. His group's method, he said, can cut that time down to a couple of weeks, thus potentially allowing for earlier treatment of a patient.

Despite the improvements to the production and design of the vaccine, Kwak said "the caveat is that the personal nature of the product still exists. We can't eliminate the fact that a novel therapeutic will be required for each patient, but we have made the cloning the gene part easy."

All told, Kwak hopes the changes his group made to the cancer vaccine will overcome the lackluster clinical data for previous DNA vaccines.

"Our strategy improves on other techniques because we have found a new mechanism of action for the myotoxin, which we are about to publish. It appears that myotoxin induces a sterile inflammation so that it doesn't just increase uptake, but it recruits antigen presenting cells to the site, which helps make the vaccine more effective."

"The manuscript describes the optimization of a vaccine therapy by delivering very localized tissue damage together with dendritic 
cell-attracting cytokines. This approach would be relatively easy and safe," said Martin Röcken, a cancer vaccine researcher who is chairman of the Department of Dermatology at Eberhard Karls University.

He said that inducing local tissue necrosis might be easier and more reproducible than the combination of an idiotype with TLR ligands. However, Röcken wasn't convinced that the toxin approach would be more effective than using TLR ligands. He said a limited number of TLR ligands were tested in the study and that their optimal mode of delivery was not investigated.

Röcken thus thinks the M.D. Anderson researchers might want to consider more preclinical studies before testing their vaccine in humans.

He told SciBX that the results in the Blood article did not represent therapeutically relevant circumstances. He said that vaccination was started a maximum of one day after tumor delivery, and he would have liked to see the effects at later time points in tumor development.

Martin Bachmann, CSO of Cytos Biotechnology AG, also is not convinced the mouse data will be reproduced in the clinic. "No one knows how this will translate to humans. DNA vaccines notoriously fail in humans. DNA vaccines always work in mice but never in humans. I personally don't understand why mouse work on DNA vaccines still can be published in good journals," he said.
Cytos' CYT004-MelQbG10, a therapeutic vaccine to treat malignant melanoma, is in Phase II testing. Cytos does not work with DNA vaccines and instead uses virus-like particles loaded with TLR ligands to stimulate the immune system.

Kwak told SciBX that the work has been patented, with one patent issued and one pending, and they are held by the NIH. The method is available for licensing.

Martz, L. SciBX 2(39); doi:10.1038/scibx.2009.1462

Published online Oct. 8, 2009

\section{REFERENCES}

1. Qin, H. et al. Blood; published online Sept. 11, 2009; doi:10.1182/blood-2009-05-219683

Contact: Larry W. Kwak, The University of Texas M.D. Anderson Cancer Center, Houston, Texas e-mail: Ikwak@mdanderson.org

2. Bendandi, M. et al. Nat. Med. 5, 1171-1177 (1999)

3. Ahonen, C. et al. J. Exp. Med. 199, 775-784 (2004)

COMPANIES AND INSTITUTIONS MENTIONED

Cytos Biotechnology AG (SIX:CYTN), Zurich, Switzerland

Eberhard Karls University, Tubingen, Germany

National Cancer Institute, Bethesda, Md.

National Institutes of Health, Bethesda, Md.

The University of Texas M.D. Anderson Cancer Center, Houston, Texas 